Rapid Reviews COVID-19

\title{
Review 2: "Obesity may hamper SARS-CoV-2 vaccine immunogenicity"
}

\section{Carlota Dobaño1}

1ISGLOBAL: Instituto de Salud Global de Barcelona, Malaria, Spain

Published on: May 07, 2021

License: Creative Commons Attribution 4.0 International License (CC-BY 4.0). 


\section{$\underline{\text { RR:C19 Evidence Scale rating by reviewer: }}$}

- Potentially informative. The main claims made are not strongly justified by the methods and data, but may yield some insight. The results and conclusions of the study may resemble those from the hypothetical ideal study, but there is substantial room for doubt. Decision-makers should consider this evidence only with a thorough understanding of its weaknesses, alongside other evidence and theory. Decisionmakers should not consider this actionable, unless the weaknesses are clearly understood and there is other theory and evidence to further support it.

*****************************************

\section{Review:}

The manuscript by Pellini and colleagues report on an analysis of antibody responses one week after the second dose of Pfizer BioNtech in a group of health care workers from Italy, reporting the titers and factors affecting them, including age, sex and BMI. They found that younger females with lower BMI have the highest responses. Although it reports potentially novel and interesting data on lower immunogenicity in obese/preobese, it needs substantial improvement in presentation and explanation of the data.

The following are revisions required:

\section{Major:}

- Indicate in the abstract that none had had pre-exposure to SARS-CoV-2. If they were not included, then there is no rationale to refer to this factor in the introduction

- Add other references in addition to Krammer et al that have been published in the last weeks about responses post-vaccination

- Provide references of peer reviewed published articles regarding RT-PCR and CLIA assays

- Specify if the test is quantitative or semi-quantitative and how is the positivity established. Did you compare with prepandemic negative samples?

- Serum was stored at $4^{\circ} \mathrm{C}$ for how long? It is normally frozen. When was the test done in relation to sample collection? Same day? 
- The reference to convalescent serum titers needs more explanation: how many, what types of patients, how long after symptoms onset, etc. Levels change largely depending on the individual and clinical presentation.

- Statistical analysis: it is not said how the adjustment by variables is done (e.g. by age) since no regression models are mentioned.

- Figures and tables need statistical information (methods and results). In figures show the individual data points, not only the boxplots. Indicate what is the boxplot showing, median and IQR? But then why do you refer to GM in the text?. Also need to indicate the threshold of positivity of the text, so that the few/only that are not seropositive (99.5\%) are visible.

- Figure 1 age: text says that it was statistically significant two-by-two comparison categories, but where is this the result of ANOVA? Mention in legend all tests used, those significant and not significant.

- Similarly for Figure 2 BMI, give all details in the legend of tests and significances

- "A correlation between BMI clases and antibody titres was noticed with $\mathrm{p}=0.02$ ": is this really correlation or T test, or ANOVA test?

- "manufacturer indicates that $80 \mathrm{AU} / \mathrm{mL}$ has a correlation of $100 \%$ with a 1:160 titre of a Plaque reduction neutralization test " - this is not acceptable, there needs to be independent evaluation of the manufacturer about tests characteristics, to ensure to what extent it reflects nAbs.

- There does not seem to be differences between pre-obesity and obesity, and the levels are very diverse in obesity, please revise the statistics and acknowledge this.

- Since we do not know the threshold for seropositivity in this report nor the protection threshold, it is now known if the vaccine would work worse in obese people. Even if levels may be lower than in people with less BMI, the levels could still be way above the protection threshold, this is not known.

Discuss this and how does obesity compromise or not responses to HepB vaccines, where a threshold of protection is known.

\section{Minor:}

- Revise use of punctuation and spaces 
- Revise use of abbreviations

- Make consistent reference to the vaccine company

- Binding antibodies method is referred twice

- Revise English and types throughout and in depth 